REVIEW

\title{
Diagnosis and disease modifying treatments in multiple sclerosis
}

\section{J Zajicek}

Postgrad Med J 2005;81:556-561. doi: 10.1136/pgmj.2004.031294

Multiple sclerosis (MS) refers to scattered areas of hardening found on sectioning central nervous system tissue of affected people, usually after many years of illness. It rarely causes early death but is the commonest cause of neurological disability among young people. Overall results from controlled trials over the past 50 years have been rather disappointing but the comparatively recent licensing of drugs such as interferon beta and glatiramer acetate has led to a reappraisal of many aspects of MS. There are now new diagnostic criteria, which encompass developments in magnetic resonance imaging. Older clinical methods of measuring disease impact are now being re-evaluated to facilitate clinical trials of the approximate 150 new products currently being developed as potential disease modifying agents. The success and failure of agents that should be effective on theoretical grounds, together with advances in neuropathology, have led to fundamental questions regarding our basic understanding of disease pathogenesis being readdressed.

Correspondence to:

Professor J Zajicek, Clinical Neurology Research, Room N16, ITTC building 1, Tamar Science Park, 1 Davy Road, Plymouth PL6 8BX, UK; john.zajicek@ phnt.swest.nhs.uk

Submitted

3 December 2004

Accepted 26 January 2005
M ultiple sclerosis (MS) has a prevalence of around 1 in 800 across most of the UK, Northern Europe, and North America. ${ }^{1}$ Some parts of Scotland have reported levels as high as 1 in 500, whereas levels are considerably lower in tropical regions and most of Asia. It is about twice as common in women than men and has a peak age of incidence around 30 years of age. Evidence from twin studies suggests that monozygotic twin concordance is around $25 \%-$ $30 \%$, compared with $3 \%-5 \%$ in dizygotic twins. ${ }^{2}{ }^{3}$ genetic component to susceptibility, compatible with a number of susceptibility genes being involved, to date, despite considerable efforts in genome screening, particular genes have not yet been identified. ${ }^{4}$ Evidence from an analysis of population migration studies suggests that exposure to as yet unidentified environmental agents before adolescence may trigger the condition in genetically predisposed people. ${ }^{5}$

Most cases of MS will start with a single episode of neurological dysfunction, most commonly in the optic nerve (optic neuritis), spinal cord (transverse myelitis), or brain stem. These episodes reflect inflammation at the relevant site within the central nervous system (CNS), and can usually be identified by areas of gadolinium Although this is consistent with a significant contrast enhancement on magnetic resonance imaging (MRI), reflecting disruption in the normally integral blood-brain barrier, with ingress of inflammatory cells. When these episodes occur, it is not possible to predict with certainty whether further episodes will occur in the future. The initial episode is therefore termed a clinically isolated syndrome (CIS), which may or may not develop into MS at a later date. If further episodes of neurological dysfunction occur, then a diagnosis of MS may be made (see diagnostic criteria below), and each episode is termed a relapse. Most people will make some recovery after each relapse, although this may be incomplete. Recovery is thought to reflect a combination of recovery in the integrity of the blood-brain barrier, a resolution of the inflammation, and repair of the disrupted myelin sheath. MS is generally considered as being an autoimmune condition, with the insulating myelin sheath being the focus of immune attack.

Unfortunately, the capacity for repair in the CNS is usually limited, and episodes of relapsing remitting MS (RR-MS), are all too often associated with a degree of permanent disability, which may become progressive with the passage of time. Although most people with MS will present with RR-MS, the bulk of this population will develop secondary progressive disease after a number of years. About 15\% of people will present with a primary progressive clinical phenotype, usually at a slightly older age and with a more equal sex distribution. We now know that even very early episodes of inflammation can be associated with significant disruption, not only to the myelin sheath and the myelin producing oligodendrocyte, but also to the underlying axons. ${ }^{67}$ This axonal loss, together with a degree of astrocyte scarring probably underlies the progressive nature of most MS. It is probable that neurons can only sustain a limited amount of inflammatory abuse, and incomplete myelin repair may remove important growth factor trophic support, and lead to a gradual loss of nerve cells, with consequent CNS atrophy measurable using MRI.

The bulk of evidence would support these suppositions, which themselves carry significant implications. For example, if correct, then very early and aggressive anti-inflammatory treatment, with ongoing immune suppression, should prevent the development of secondary

Abbreviations: MS, multiple sclerosis; MRI, magnetic resonance imaging; CNS, central nervous system; CIS, clinically isolated syndrome; RR-MS, relapsing remitting multiple sclerosis 
progressive disease. There is currently no proof to substantiate this hypothesis. Furthermore, in primary progressive disease, where comparatively little cerebral inflammation is apparent, anti-inflammatory treatments seem to be ineffective. Some people have gone so far as to question whether MS related inflammation is in fact a secondary, rather than a primary phenomenon. In other words, could some forms of MS be due to primary axonal or oligodendrocyte degeneration?

Some re-appraisal of the traditional homogenous nature of MS has occurred after recent neuropathological studies. The problem with much of the older MS literature has been that it is based on tissue from advanced cases. Once the inflammatory process has been occurring for many years, it is probable that many secondary and tertiary reactions are taking place. Trying to disentangle primary disease under these circumstances is extremely difficult. More recently there have been concerted attempts at analysing MS tissue from people very early in the disease process such as after sudden death from an unrelated cause, or where a biopsy has been performed in cases of diagnostic uncertainty. Results from such studies have suggested that MS pathology may not be the same in all cases. Four major disease types have been described, types I and II being largely inflammatory and immune mediated, whereas types III and IV seem to be more typical of primary cell death, rather than immunologically driven. ${ }^{8}$ Interestingly all lesions from any individual patient seem to take the same form, but people differ in their disease subtype. There has also been a suggestion that apoptotic cell death of oligodendrocytes may underlie all early lesions, so phagocytosis of disrupted myelin may be a secondary event. ${ }^{9}$ If these results can be applied more widely, they will have profound implications for our understanding of MS. Rather than accepting everyone has the same basic disease process, we may need to direct specific treatments to particular disease subtypes, as well as investigating ways of influencing the cell death programmes of oligodendrocytes.

\section{CRITERIA FOR MAKING THE DIAGNOSIS OF MS}

There is no single diagnostic test that is specific to MS, and making a diagnosis still rests on confirmation of dissemination of CNS inflammation, both in time and position within the CNS. The important change in diagnostic criteria is the inclusion of MRI as a means of assisting in the identification of new lesions, rather than just relying on clinical evidence for lesion dissemination. ${ }^{10}$ In other words, to make a diagnosis of MS after a clinically isolated syndrome, there must be objective evidence of a second lesion to show dissemination in space, which could be provided by an MRI scan. When the presentation is of insidious neurological progression, spinal fluid analysis demonstrating electrophoretic oligoclonal IgG bands, different from any bands in serum, is necessary to have a convincing diagnosis of primary progressive MS.

It is important to emphasise that although cranial MRI appearances may be suggestive of MS, they are not specific enough to make a diagnosis in the absence of appropriate clinical support. Depending on which criteria are adopted, specificity rates of around $75 \%$ are the best obtainable. ${ }^{11}$ In addition, the diagnosis of MS can only be made if no better alternative explanation for the neurological features can be found. The supreme sensitivity of MRI can lead to pitfalls for the unwary. It is not uncommon for MRI to be performed without adequate thought about potential implications if abnormalities are found. For example, the identification of non-specific lesions in apparently well people is an established phenomenon, particularly with advancing age over 50. Often knowledge of an abnormal MRI serves only to create anxiety in the patient, and unless the requesting physician knows how to deal with results of unexpected findings on MRI, and has a very good reason for requesting the scan, then MRI should not be undertaken lightly.

Some controversy also exists around the communication of a potential diagnosis of MS to patients. In conditions such a optic neuritis, where there is an established risk of developing MS, which may be as high as $70 \%-80 \%$ over 25 years, there is evidence that if lesions are seen on MRI at presentation, the risk is higher. ${ }^{12}$ Conversely a normal cranial MRI seems to confer lower risk of subsequent MS development (around $20 \%$ after 14 years $^{12}$ ). The precise figures are difficult to define, as long term studies with full patient retention are difficult to conduct. None the less, the issue is whether this potential diagnosis should be conferred to patients at initial presentation. Recent guidelines from the UK National Institute for Health and Clinical Excellence (NICE) ${ }^{13}$ recommend that a person should be informed of the potential diagnosis of MS, as soon as a diagnosis of MS is considered reasonably likely, unless there are overwhelming patient centred reasons for not doing so. In addition, it is recommended that an ophthalmologist should communicate the potential diagnosis of MS after making the diagnosis of optic neuritis. These recommendations could also create anxiety in patients, particularly if there are delays in getting more specialist opinions. This is particularly difficult when people present with episodes of numbness and tingling. Making decisions about whether to investigate at all, let alone whether to convey potential devastating diagnoses can be difficult for doctors. Overall this author would endorse the communication of potential information and appropriate investigation of patients, but only if the doctor concerned has the necessary knowledge to be able to deal with any consequences.

\section{CHANGING THE DISEASE COURSE IN MS}

One of the few predictable features of MS is that it is unpredictable, particularly early in the condition. To ascertain whether any treatment is having an impact on any patient's MS, ideally we would be able to predict what would happen with and without that particular treatment. Current treatment options are a long way from this goal, and although certain drugs have been labelled as being disease modifying (a term used particularly by the pharmaceutical industry), there are no long term studies that show a reduction in the incidence of secondary progression. Other terms may be more appropriately used, such as immunomodulatory or neuroprotective, but only where the data support such terminology.

Most clinical trials have been designed using data derived from large natural history cohorts using a measurement scale known as the expanded disability status scale (EDSS). These data can also be used to advise patients in generic terms about prognosis. So, for example, being a woman, with few relapses, mainly sensory and optic nerve symptoms, little to find on examination, and with no coordination problems, are all good prognostic factors. Conversely, being male, with a mainly progressive pattern, with motor and coordination problems, and persistent abnormalities on neurological examination at an early stage of the condition, are all poorer prognostic factors. Attempting to individualise population or large trial data is fraught with problems, but none the less a necessary requirement in trying to tailor treatment for individual patients and in counselling people about treatment options.

It is often difficult for clinicians outside of a particular disease area to appreciate the complexities and idiosyncrasies of any particular measurement tool. The EDSS is disease specific and ranges from 0 (normal on examination) to 10 (dead). The first four points are based on neurological examination findings, scores from 4-7 based on mobility, and 
scores above this based on degree of dependence. The scale is not linear, and changes of one point at lower levels on the scale do not equate to single point changes at higher scores. Patients spend different amounts of time at each level, there is no measurement of cognition (which unfortunately is commonly affected in MS), and overall the scale is very insensitive. Despite these shortcomings (and there are others), it is this scale that is the basis of virtually all trials of potential disease modification in MS. Better measurement tools have been developed, and we all look forward to the day that generic scales of physical disability, psychological and social wellbeing can be used across all medical conditions.

\section{INTERFERON BETA AND GLATIRAMER ACETATE}

There is now considerable clinical experience of using these drugs in routine clinical practice. We know from the pivotal studies that these compounds share similar levels of clinical efficacy, although there are two major types of interferon beta ( $1 \mathrm{a}$ and $\mathrm{lb}$ ), and three different interferon beta products $^{14-17}$ as well as glatiramer acetate. ${ }^{18}$ When used in ambulant patients with RR-MS, and a relapse rate of around one per year for the previous two to three years (the average relapse rate of RR-MS being one every two years), patients may expect a reduction in relapses of about $30 \%$. Another way of putting this statistic is that if the treatment works and someone is having a relapse a year, over a three year period, a patient with RR-MS might expect two relapses instead of three. The evidence would suggest that these drugs do not work in progressive disease, unless there are concomitant relapses. ${ }^{19}$

There is more controversy over the long term effects of these drugs and whether they truly have an impact on disability. A recent Cochrane review of interferon beta in $\mathrm{MS}^{20}$ identified seven trials that met the criteria of double blind, placebo controlled, randomised trials of RR-MS. Although 1215 patients were included in this review, only $919(76 \%)$ contributed to the results concerning exacerbations and progression of the disease at two years. Specifically interferon significantly reduced the occurrence of exacerbations $(\mathrm{RR}=0.80,95 \%$ CI 0.73, 0.88, p <0.001) and progression of the disease $(\mathrm{RR}=0.69,95 \%$ CI $0.55,0.87$, $\mathrm{p}=0.002$ ) two years after randomisation. However, the clinical course and assignment of drop outs was essential to the demonstration of efficacy, most conspicuously concerning the effect of the drug on disease progression. Hence, if interferon treated patients who dropped out were deemed to have progressed (worst case scenario) the significance of these effects was lost $(\mathrm{RR}=1.31, \mathrm{CI} 0.60,2.89, \mathrm{p}=0.5)$. A further recent Cochrane review of glatiramer acetate concluded that there was little evidence of any beneficial effect on the main outcome measures in MS (both disease progression and risk of clinical relapses). ${ }^{21}$ These drugs have been controversial mainly because of their cost in relation to the size of any beneficial effect. There is fierce global commercial competition for the available market, and publications should be interpreted on the basis of scientific content, with an understanding of the background interests. For example there is an ever-expanding literature on the significance of anti-interferon antibodies in relation to lack of clinical efficacy. This literature has generated more heat than light, and may serve to cloud interpretation of other, more important data. Part of the problem in the interpretation of data from immunomodulatory studies in MS is that the most studies have only been placebo controlled for two years. Lessons can be gleaned from the Mayo Clinic-Canadian cooperative study of sulfasalazine in MS here. ${ }^{22}$ This was a three year study, which showed no significant treatment effects at three years, although there were effects at two years. Thus, if this study were only of two years duration, it would have been judged to be a successful treatment, which it is not. In the UK the Association of British Neurologists has produced guidelines ${ }^{23}$ on the use of interferon beta and glatiramer acetate, which are a useful summary of available evidence and recommended practice.

Two further points are worth mentioning. Firstly, although there have been two published studies of interferon beta in $\mathrm{CIS}^{24}{ }^{25}$ the results of both studies are consistent with previous information on reduction in relapse rate. It is not surprising that if these compounds reduce relapses, then we would expect to see a prolongation of time between CIS and development of MS in the treatment group. This is not powerful evidence for treating CIS at the earliest opportunity, and we desperately need longer term studies to establish whether early treatment really does have an impact on long term clinical disease course. Secondly, these compounds (interferon beta much more than glatiramer acetate) do have large effects on aspects of MRI at follow up. The problem here is that the clinical correlation of disability with cranial MRI is not good, and if we are not careful, the tendency to treat scans rather than patients becomes manifest. If surrogate outcome measures are reliable and clinically well correlated, (for example, tumour mass in cancer treatment), then it is reasonable to accept surrogate measures of treatment effect. This is not yet the case in MS, and ultimately we need both better clinical measures and better surrogate measures that accurately reflect the most important clinical aspects of the condition from the patient and carer perspective.

\section{THE UK RISK SHARING SCHEME}

The cost of interferon beta and glatiramer acetate treatment led to these drugs being early targets for the newly formed UK National Institute for Health and Clinical Excellence (NICE), whose role includes a cost effectiveness analysis of new treatments and interventions, to help decide whether treatments are effective and affordable to the UK NHS. Their report from 2002 concluded that these treatments were not cost effective and should not be used routinely in the NHS in England and Wales, unless costs could be reduced. Not surprisingly, the pharmaceutical industry, together with a vocal patient group argued strongly against this conclusion, which led to an exploration of alternative ways of reducing the cost and also the risk of these treatments not being cost effective. The UK "Risk Sharing Scheme" was essentially developed to enable patient access to treatment at reduced cost, with monitoring of patient disability over time, with the eventual aim of further adjusting prices, depending on data generated by the monitoring scheme. This innovative solution to the NICE report has been criticised, ${ }^{26}$ particularly for the lack of scientific rigour of the monitoring scheme, and the missed opportunity for conducting well designed independent clinical trials rather than simply monitoring patients without a control group. Rather than spending tens of millions of pounds on treatments with questionable long term benefit, a fraction of these costs could have generated

$A B N$ criteria to receive interferon beta in relapsing remitting $\mathrm{MS}$

- Able to walk independently, at least 10 metres with assistance

- At least two clinically significant relapses in the previous two years

- Age 18 or older

- No contraindications, for example, no monoclonal gammopathy 
reliable scientific data on which rational decisions about the place of these drugs in the long term management of MS could have been made. It is now most unlikely that independent long term clinical trials of these compounds, or any other compounds in which a placebo group is involved, can be conducted in RR-MS. Lessons need to be learnt from this saga, and hopefully if more investment in independent clinical trials occurs, linked with a speedier early evaluation of treatments, then similar episodes may be avoided in the future. There is good evidence that patients benefit from taking part in clinical trials, even if receiving placebo treatments. Every opportunity must be taken to obtain high quality scientific data if real disease modifying treatments are to be identified with confidence.

\section{OTHER IMMUNOMODULATORY DRUGS}

There is a range of drugs currently either being used to treat MS, or in development. A recent estimate of around 150 new compounds in the production pipeline is probably accurate. Most compounds are immunomodulatory in some way, ranging from non-specific immunosupressants such as azathioprine and mitoxantrone, through more specific monoclonal antibody treatments such as alemtuzumab (Campath $1 \mathrm{H})^{27}$ and natalizumab (Antegren), ${ }^{28}$ to attempts at blocking antigen presentation at the immunomological trimolecular complex between $\mathrm{T}$ cells and antigen presenting cells. ${ }^{29}$ A full consideration of all compounds in development is beyond the scope of this review, but some general points are worth considering.

Some of the compounds currently being tested are extremely powerful, both on clinical and MRI parameters. However, none has yet proved to be without significant side effects. For example, alemtuzumab is very effective at abolishing new MRI activity and most clinical relapses, but has a $30 \%$ risk of thyrotoxicosis at the doses previously used. If these products are to make a useful transition from clinical trials to routine practice, they will almost certainly need to be targeted to those patients in whom they are most likely to be useful. This means that in patients likely to have a benign disease course, milder treatments with fewer side effects may be a more sensible option. On the other hand, patients destined for a catastrophic disease may warrant the most powerful treatment options. The difficulty comes in spotting which patients fall into each category, and we simply do not have accurate methods of predicting disease course with any certainty. Once again, treatment algorithms should be tested in clinical trials to provide clinicians with appropriate decision making tools.

Another issue relates to when to use these compounds. As described above, if we believe that MS is fundamentally an autoimmune disorder, with damage sufficient to trigger the processes necessary for secondary degeneration occurring early in the disease course, then strong treatment given early is the most logical step. We still need evidence to back up our theories, and in our present information vacuum, there is a strong argument for appropriately counselling patients about

\section{Available types of interferon beta for treating} MS

- Interferon beta $1 \mathrm{~b}$ (Betaferon)-for subcutaneous injection, $300 \mu \mathrm{g}$ alternate days

- Interferon beta la (Avonex)-for intramuscular injection $30 \mu \mathrm{g}$ once weekly

- Interferon beta la (Rebif) - for subcutaneous injection 22 or $44 \mu \mathrm{g}$ three times/week clinical risk. In a notoriously unpredictable disease, do they wish to wait and see what happens to them, by which time immune intervention therapies may have become ineffective, or do they wish to take a long term risk with comparatively untried treatments in a gamble that their disease is going to get worse?

A further issue revolves around how accurately we are able to predict the behaviour of the immune system if we target one component of it. Most of the animal and experimental data would suggest that tumour necrosis factor $\alpha(\mathrm{TNF} \alpha)$ is harmful in $\mathrm{MS}^{30}{ }^{31}$ yet a treatment aimed at blocking TNF $\alpha$ action (Lenercept) actually made the disease worse. ${ }^{32}$ Most treatments effective in animal models of MS have not made the transition to human use, perhaps illustrating the complexity of nature and our inadequate understanding of damage and repair mechanisms. There is also considerable redundancy in the immune system, so for example, there are several adhesion molecules and chemokines involved in migration of white cells across the blood-brain barrier into the CNS. Targeting any single molecule may fail if there are alternatives ready to step into the immunological breach when one molecule is blocked. Ultimately there is no substitute for good, preferable long term, clinical trials and until we have more evidence, it is very difficult to make recommendations for everyday practice. Consideration of which drug to use when and in whom is best made by a neurologist with expertise of using these drugs, after appropriate patient counselling.

Unfortunately, the lack of convincingly effective treatments, especially later in the disease course means that patients may become desperate for treatments based on the latest anecdotal information. The history of MS treatment is littered with treatment failures after huge initial enthusiasm. Recent results showing the size of placebo effects in MS trials only serves to illustrate this point. ${ }^{33}$ Such placebo responses, possibly improved by the increased care and attention given to participants in clinical trials, may persist for many months, if not years, and experienced clinicians learn to become immune to the power of anecdote.

\section{TREATMENTS FOR PROGRESSIVE DISEASE}

Unfortunately there is no good evidence for any drug having an effect in progressive forms of MS in the absence of relapses. Many clinicians, often after heartfelt discussion with patients, have tried powerful immunotherapies at this stage of the illness, but with little, if any success. This is compatible with the hypothesis that if immunotherapies are going to work, they must be used early in the illness. There is mounting evidence from natural history studies that once walking starts to become affected in people with MS, then there is a steady decline in physical functioning, which at present cannot be halted. A degree of optimism can be given to patients by telling them that most people with MS will not die from their disease, and disability does tend to stabilise over time, but it is impossible to predict at what point that will occur, or how disabled people will be when things settle down.

In this environment, there is now a massive swelling of interest in strategies aimed at slowing down progressive disease, so called neuroprotective therapies. Many compounds may have such a potential action, including drugs blocking the excitotoxic mechanisms of glutamate action, treatments to reduce free radical damage (including antioxidants), and trophic therapies designed to provide growth factors to damaged nerve cells. Once again lessons can be learnt from clinical trials in other disease areas. For example, the DATATOP study ${ }^{34}$ examining the use of selegiline in the treatment of Parkinson's disease was confounded by the symptomatic effect of the drug. In other words people felt 
better receiving active medication because of a symptomatic rather than a fundamental neuroprotective effect of the drug. This is a potentially significant problem with any treatment where a disability scale is used as a primary trial outcome, yet such measures are crucial to lending clinical credence to any positive results from surrogate measures such a laboratory tests or MRI. The future lies in developing rating scales that are stable and linear, which can be used to disentangle the complex relation between symptoms and disability. Ideally surrogate measures will be used in parallel with such clinical scales and can also be used to illuminate pathological mechanisms in chronic disease.

\section{CONCLUSIONS}

This is an exciting time in MS care and research. A slow but steady array of drugs is becoming available that may be useful in the battle against this potentially disabling condition. Overall, the increased availability of drugs such as interferon beta and glatiramer acetate has increased resources and attention towards MS care. Yet there remain a significant number of challenges in future treatment strategies. Accurate diagnosis needs to be combined with some estimate of prognosis that should assist in making treatment decisions. Increasing availability of information needs to be combined with education of both health professionals and patients to the importance of developing research strategies to assist in answering the difficult questions. Finally, there must be a unity of purpose from everyone involved with MS, to ensure precious resources are directed at better treatments rather than commercial priorities.

\section{MULTIPLE CHOICE QUESTIONS (ANSWERS AT END OF REFERENCES)}

1. When a person presents with a clinically isolated syndrome, and has a normal cranial MRI, what is the 14 year risk of developing MS?
(A) $10 \%$
(B) $20 \%$
(C) $50 \%$
(D) $60 \%$
(E) $80 \%$.

2. Which of the following treatments are effective for primary progressive MS?

(A) glatiramer acetate

(B) interferon beta la high dose

(C) interferon beta la low dose

(D) mitoxantrone

(E) none of these.

3. What is the overall likelihood of relapse reduction using interferon beta if someone with MS had been having one relapse per year for the previous two years?
(A) $5 \%$
(B) $10 \%$
(C) $30 \%$
(D) $50 \%$
(E) $70 \%$

4. What is the twin concordance rate for MS in monozygotic twins?

(A) $5 \%$
(B) $10 \%$

(C) $25 \%$

(D) $50 \%$

(E) $100 \%$

5. What is the highest overall prevalence rate of MS in parts of Scotland?
(A) 1 in 200
(B) 1 in 300
(C) 1 in 800
(D) 1 in 500
(E) 1 in 1000
Funding: none.

Conflicts of interest: none.

\section{REFERENCES}

1 Fox CM, Bensa S, Bray l, et al. The epidemiology of multiple sclerosis in Devon: a comparison of the new and old classification criteria. J Neurol Neurosurg Psychiatry 2004;75:56-60.

2 Willer CJ, Dyment DA, Risch NJ, et al. Twin concordance and sibling recurrence rates in multiple sclerosis. Proc Natl Acad Sci U S A 2003; 100: 12877-82.

3 Mumford CJ, Wood NW, Kellar-wood H, et al. The British Isles survey of multiple sclerosis in twins. Neurology 1994;44:11-15.

4 Sawcer S, Maranian M, Setakis E, et al. A whole genome screen for linkage disequilibrium in multiple sclerosis confirms disease associations with regions previously linked to susceptibility. Brain 2002; 125:1337-47.

5 Compston DAS. Distribution of multiple sclerosis. In: Compston A, Lassmann H, McDonald I, et al, eds. McAlpine's multiple sclerosis. 3rd ed. London: Churchill Livingstone, 1998:63-94.

6 Charcot JM. Lecons sur les maladies du systeme nerveux faites a la Salpetriere. Vol 1. 4th ed. Paris: 1880.

7 Trapp BD, Peterson J, Ransohoff RM, et al. Axonal transection in the lesion of multiple sclerosis. N Engl J Med 1998;338:278-85.

8 Lucchinetti C, Bruck W, Parisi J, et al. A quantitative analysis of oligodendrocytes in multiple sclerosis lesions. A study of 113 cases. Brain 1999; 122:2279-95.

9 Barnett MH, Prineas JW. Relapsing and remitting multiple sclerosis: pathology of the newly forming lesion. Ann Neurol 2004;55:458-68.

10 McDonald WI, Compston A, Edan G, et al. Recommended diagnostic criteria for multiple sclerosis: guidelines from the international panel on the diagnosis of multiple sclerosis. Ann Neurol 2001;50:121-7.

11 Tintore M, Rovira A, Martinez MJ, et al. Isolated demyelinating syndrome: comparison of different MR imaging criteria to predict conversion to clinically definite multiple sclerosis. Am J Neuroradiol 2000;21:702-6.

12 Brex PA, Ciccarelli O, O'Riordan Jl, et al. A longitudinal study of abnormalities on MRI and disability in multiple sclerosis. N Engl J Med 2002;346: 158-64

13 NICE. Full guidance on the use of beta-interferon and glatiramer for the treatment of multiple sclerosis. London: NICE, 2002: 32. http:// www.nice.org.uk/page. $a s p x ? o=27635$.

14 Duquette $P$, Girard M, Despault $L$, et al. Interferon beta- $1 \mathrm{~b}$ is effective in relapsing-remitting multiple- sclerosis - clinical-results of a multicenter, randomized, double- blind, placebo-controlled trial. Neurology 1993;43:655-61.

15 Duquette $\mathrm{P}$, Despault L, Knobler RL, et al. Interferon beta- $1 \mathrm{~b}$ in the treatment of multiple sclerosis: Final outcome of the randomized controlled trial. Neurology 1995;45: 1277-85.

16 Jacobs LD, Cookfair DL, Rudick RA, et al. Intramuscular interferon beta-1 a for disease progression in relapsing multiple sclerosis. The Multiple Sclerosis Collaborative Research Group (MSCRG). Ann Neurol 1996;39:285-94.

17 PRISMS (Prevention of Relapses and Disability by Interferon beta-1 a Subcutaneously in Multiple Sclerosis) Study Group. Randomised double-blind placebo-controlled study of interferon beta- $1 \mathrm{a}$ in relapsing/remitting multiple sclerosis. Lancet 1998;352:1498-504.

18 Johnson KP, Brooks BR, Cohen JA, et al. Copolymer 1 reduces relapse rate and improves disability in relapsing-remitting multiple sclerosis: results of a phase III multicenter, double-blind placebo-controlled trial. The Copolymer Multiple Sclerosis Study Group. Neurology 1995;45:1268-76.

19 European Study Group. Placebo-controlled multicentre randomised trial of interferon beta-1b in treatment of secondary progressive multiple sclerosis. European Study Group on interferon beta-1b in secondary progressive MS. Lancet 1998:352:1491-7.

20 Rice G, Incorarvaia B, Munari L, et al. Interferon in relapsing-remitting multiple sclerosis. Cochrane Library. Issue 4. Oxford: Update Software, 2001.

21 Munari L, Lovati R, Boiko A. Therapy with glatiramer acetate for multiple sclerosis. Cochrane Library. Chichester: Wiley, 2004.

22 Noseworthy JH, O'Brien P, Erickson BJ, et al. The Mayo Clinic-Canadian cooperative trial of sulfasalazine in active multiple sclerosis. Neurology 1998:51:1342-52. 
23 Association of British Neurologists. Guidelines for the use of beta interferons and glatiramer acetate in multiple sclerosis. London: ABN, 2001. http:// www.theabn.org/downloads/msdoc.pdf.

24 Jacobs L, Beck R, Simon J, et al. Intramuscular interferon beta- la therapy initiated during a first demyelinating event in multiple sclerosis. N Engl J Med 2001;343:898-904.

25 Comi G, Filippi M, Barkhof F, and the Early Treatment of Multiple Sclerosis Study Group. Effect of early IFN treatment on conversion to definite MS: a randomised study. Lancet 2001;357:1576-82.

26 Sudlow CLM, Counsell CE. Problems with the UK government's risk sharing scheme for assessing drugs for multiple sclerosis. BMJ 2003;326:388-92.

27 Coles AJ, Wing M, Smith S, et al. Pulsed monoclonal antibody treatment and autoimmune thyroid disease in multiple sclerosis. Lancet 1999:354:1691-5.

28 Miller DH, Khan OA, Sheremata WA, et al. A controlled trial of natalizumab for relapsing multiple sclerosis. N Engl J Med 2003;348:15-23.

29 Hohlfeld R. Biotechnological agents for the immunotherapy of multiple sclerosis. Principles, problems and perspectives. Brain 1997;120:865-916.
30 Selmai K, Raine CS. Tumour necrosis factor mediates myelin and oligodendrocyte damage in vitro. Ann Neurol 1988;23:339-46.

31 Zajicek J, Wing M, Scolding NJ, et al. Interactions between oligodendrocytes and microglia, a major role for complement and tumour necrosis factor in oligodendrocyte adherence and killing. Brain 1992;115:1611-31.

32 Lenercept Multiple Sclerosis Study Group. TNF neutralization in MS; results of a randomized, placebo controlled multicentre study. Neurology 1999;53:457-65.

33 Zaiicek J, Fox P, Sanders H, et al. The use of cannabinoids for the treatment of spasticity and other symptoms related to multiple sclerosis - results of a multicentre randomised placebo-controlled trial (the CAMS study). Lancet 2003;362:1517-26

34 Stocchi F, Olanow CW. Neuroprotection in Parkinson's disease: clinical trials. Ann Neurol 2003;53(suppl 3):S87-97, S97-9.

\section{ANSWERS}

1. (B); 2. (E); 3. (C); 4. (C); 5. (D). 